\title{
ASPECTOS DA BIOLOGIA DE LUTZOMYLA DO COMPLEXO SQUAMIVENTRIS (LUTZ \& NEIVA,1912)(DIPTERA, PSYCHODIDAE) EM CONDIÇÕES NATURAIS, NA CIDADE DE GOIÂNIA-GOIÁS
}

Maria Elisa Santos Dourado Carvalho * Heloisa Aparecida Machado Naves **

\section{RESUMO}

Coletaram-se 201 exemplares de Lu.squamiventris em capturas realizadas com isca humana em solo e copa de árvore, isca animal e armadilhas luminosas (Shannon e CDC) nos horários matutino, vespertino e noturno, na Chácara Naves, nos anos de 1987, 1988, 1991 e 1992.

O maior número de exemplares foi obtido no ano de 1992, seguindo-se 1988 e 1991.

Sua maior ocorrência foi nos meses de janeiro, fevereiro e março.

Isca humana no solo foi a preferida por esta espécie $(75,12 \%)$ seguindo-se armadilha de Shannon (23,88\%): isca animal e armadilha CDC $(0,49 \%)$. Não foi capturado nenhum exemplar em isca humana na plataforma.

Com relaçåo à frequência horária observou-se uma maior ocorrência do flebótomo entre 19 e 20 horas, obtendo-se neste intervalo $46,26 \%$ dos exemplares.

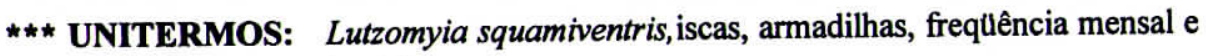
horária.

\section{INTRODUÇÃO}

As leishmanioses constituem endemias importantes em Goiás. De 1963 a 1964, BARBOSA et al. descreveram 565 casos autóctones de leishmaniose tegumentar americana, em vários municípios goianos e de 1985 a 1986, a FNS registrou um total de 905 casos. NASCIMENTO (1986), faz ampla revisão sobre leishmaniose em nosso Estado mostrando os reduzidos dados sobre os estudos dos transmissores.

$O$ aparecimento de novos casos de leishmaniose tegumentar em vários municípios chamou atençao da equipe de entomologia do IPTSP para a necessidade de

- Bióloga do Depto. de Parasitologia-IPTSP-UFG C.P. 131 Goiânia-Goiás

-. Professora do Depto. de Parasitologia-IPTSP-UFG C.P. 131 Goiânia-Goiás

-. Comunicação pessoal

Recebido para publicação em 04/08/94 
CARVALHO,M.E.S.; NAVES.J.A.M. Aspectos da Biologia de Lutzomyia do Complexo squamiventris (Lutz \& Neiva, 1912) (Diptera, Psychodidae) em Condiçð̃es Naturais, na cidade de Goiânia-Goiás. Rev.Pat.Trop.,23 ( 2 ):229-234,jul./dez.1994

melhores estudos da fauna flebotomínica nao apenas em Goiás, mas principalmente em Goiânia.

Os únicos estudos realizados sobre flebotomíneos em nosso Estado foram os de BARRETO; (1946); MARTINS et al. (1962,1964 e 1975); COELHO et al. (1965); LUSTOSA et al. (1986); CARVALHO et al. (1989); NAVES et al. (1991); CARVALHO et al. (1992); NAVES et al. (1992); CARVALHO et al. (1993) e NAVES et al. (1993).

Os flebótomos foram identificados segundo FORATTINI (1973), GALATI (1990), MARTINS, WILLIANS \& FALCÃO (1978), RYAN (1986) e YOUNG \& FAIRCHILD, 1974.

\section{MATERIAIS E MÉTODOS}

A área estudada localiza-se na região norte de Goiânia, próxima ao Bairro Fama, Setor Meia Ponte e Vila Itatiaia. A estação de coleta situa-se em mata de Interflúvio, que se caracteriza pela queda não uniforme das folhas, principalmente nos períodos mais secos do ano. Devido à queda de folhas, ramos, flores, frutos, cascas e troncos, o solo fica descoberto o que é importante na reciclagem de nutrientes.

À margem da mata, encontram-se casas permanentemente habitadas, piscinas, e criações de animais.

Realizaram-se 79 capturas nos anos de 1987, 1988, 1991 e 1992, totalizando 766 horas de coleta.

Para observar a referência alimentar utilizou-se de canídeo (Canis), galináceo (Gallus) e suíno (Scrofa). Com relação à isca humana, as coletas foram realizadas por duas pessoas que no solo e plataforma funcionavam como isca e ao mesmo tempo como capturador.

Com relação às armadilhas luminosas utilizou-se de Shannon em mata, CDC em mata, galinheiro, pocilga e plataforma.

\section{RESULTADOS}

Obtiveram-se 201 exemplares de Lutzomya squamiventris, na Chácara Naves, Goiânia-Goiás, nos anos de 1987, 1988, 1991 e 1992.

Sua maior ocoorência deu-se no ano de 1992 (76,11\% dos exemplares) seguindo-se $1988(22,38 \%)$ e $1991(0,99 \%)$. As capturas realizadas em 1987 foram negativas para esta espécie. (TABELA 1).
CARVALHO,M.E.S.; NAVES.J.A.M. Aspectos da Biologia de Lutzomyia do Complexo squamiventris (Lutz \& Neiva, 1912) (Diptera, Psychodidae) em Condiçð̃es Naturais, na cidade de Goiânia-Goiás. Rev.Pat.Trop.,23 ( 2 ):229-234,jul./dez.1994

TABELA 1. Número mensal de Lu.squamiventris coletado em Goiânia-Goiás.

\begin{tabular}{|c|c|c|c|c|c|c|c|c|c|c|c|c|c|}
\hline \multirow[t]{2}{*}{ Anos } & \multicolumn{12}{|c|}{ Meses } & Total \\
\hline & Jan & Fev & Mar & Abr & Mai & Jun & Jul & Ago & Set & Out & Nov & Dez & \\
\hline 1987 & - & - & - & - & - & - & - & - & - & - & - & - & \\
\hline 1988 & - & 03 & 42 & - & - & - & - & - & - & - & - & - & 45 \\
\hline 1991 & - & - & - & - & - & - & - & - & - & - & 02 & - & 02 \\
\hline 1992 & 136 & 14 & - & - & 02 & - & 01 & - & 01 & - & - & - & 153 \\
\hline TOTAL & 136 & 17 & 42 & - & 02 & - & 01 & - & 01 & - & 02 & - & 201 \\
\hline
\end{tabular}

Observou-se uma maior incidência da mesma no mês de janeiro $(67,66 \%)$ seguindo-se março $(20,89 \%)$ e fevereiro $(8,45 \%)$.

As coletas nos meses de abril, agosto, outubro e dezembro foram negativas para esta espécie.

Com relação a iscas e armadilhas utilizadas, verificou-se maior eficácia para isca humana no solo $75,12 \%$ dos exemplares. Obteve-se em armadilha de Shannon 23 $88 \%$ em isca animal e CDC $0,49 \%$.

Nenhum exemplar de Lu.squamiventris foi coletado em plataforma. (TABELA 2).

TABELA 2. Número mensal de Lu.squamiventris coletado simultaneamente em isca humana ao nivel do solo e em plataforma, isca animal e armadilhas luminosas nos anos de 1987,1988,1991 e 1992.

\begin{tabular}{|c|c|c|c|c|c|c|c|c|c|c|c|c|c|}
\hline \multirow{2}{*}{$\begin{array}{l}\text { Iscas e } \\
\text { armadilhas }\end{array}$} & \multicolumn{11}{|c|}{ Meses } & \multicolumn{2}{|r|}{ Total } \\
\hline & Jan & Fev & Mar & $\mathrm{Abr}$ & Mai & Jun & Jul & Ago & Set & Out & Nov & Dez & 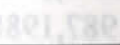 \\
\hline $\begin{array}{l}\text { Isca Humana } \\
\text { (solo) }\end{array}$ & 95 & 14 & 40 & - & 01 & - & 01 & - & - & - & - & - & 151 \\
\hline $\begin{array}{l}\text { Isca Humana } \\
\text { (plataforma) }\end{array}$ & - & - & - & - & - & - & - & - & - & - & - & - & - \\
\hline Isca animal & - & - & - & - & - & - & - & - & 01 & - & - & - & 01 \\
\hline Shannon & 41 & 02 & 02 & - & 01 & - & - & - & - & - & 02 & - & 48 \\
\hline $\mathrm{CDC}$ & - & 01 & - & - & - & - & - & - & - & - & - & - & 01 \\
\hline TOTAL & 136 & 17 & 42 & - & 02 & - & 01 & - & 01 & - & 02 & - & 201 \\
\hline
\end{tabular}


CARVALHO,M.E.S; NAVES.J.A.M. Aspectos da Biologia de Lutzomyia do Complexo squamiventris (Lutz \& Neiva, 1912) (Diptera, Psychodidae) em Condiçðes Naturais, na cidade de Goiânia-Goiás. Rev.Pat.Trop.,23 ( 2 ):229-234,jul./dez.1994

Com relação à frequiência horária, observou-se ocorrência de exemplares a partir do crepúsculo vespertino. Os horários mais produtivos foram: 19-20 horas $46,26 \%)$ : $18-19$ h. (29,85\%); $20-21$ h. $(13,93 \%)$ e $17-18 \mathrm{~h} .,(9,45 \%)$. Nenhum espécime foi coletado entre 9-16h. (TABELA 3).

TABELA 3. Frequência horária de Lu.squamiventris coletado em Goiânia, nos anos de 1986,1987,1991 e 1992.

\begin{tabular}{|c|c|c|c|c|c|c|c|c|c|c|c|c|c|}
\hline \multirow[t]{2}{*}{ Horário } & \multicolumn{12}{|c|}{ Meses } & \multirow[t]{2}{*}{ Total } \\
\hline & Jan & Fev & Mar & Abr & Mai & Jun & Jul & Ago & Set & Out & Nov & Dez & \\
\hline $16-17$ & - & - & - & - & - & - & - & - & 01 & - & - & - & 01 \\
\hline $17-18$ & - & 14 & 05 & - & - & - & - & - & - & - & - & - & 19 \\
\hline $18-19$ & 35 & 03 & 20 & - & 02 & - & - & - & - & - & - & - & 60 \\
\hline $19-20$ & 82 & - & 10 & - & - & - & 01 & - & - & - & - & - & 93 \\
\hline $20-21$ & 19 & - & 17 & - & - & - & - & - & - & - & 02 & - & 28 \\
\hline TOTAL & 136 & 17 & 42 & - & 02 & - & 01 & - & 01 & - & 02 & - & 201 \\
\hline
\end{tabular}

SUMMARY

Biology aspects of Lutzomyia squamiventris (Lutz \& Neiva, 1912) (Diptera, Psychodidae) in Natural conditions in Goiânia city, Goiás.

201 exemplaries of Lu.squamiventris were collected in captures realized with human baits in ground and on top tree, animal bait, and luminous traps (Shannon and CDC), in periods: morning, afternoon and night, in Naves Farm, in yars $1987,1988,1991,1992$. and 1991.

The major amount of exemplaries was obtained in 1992, followed by 1988

Its major ocurrence was in January and March.

This type prefered human bait in ground $(75,12 \%)$ then Shannon's trap (23,88\%); animal bait and CDC trap $(0,49 \%)$.

Related to the time frequency, it was observed major ocurrence of flebotomus beteween 07:00pm and 08:00pm; 46,26\% of the exemplaries.

KEYWORDS: Lutzomyia squamiventris, baits,traps,month and time frequency,
CARVALHO,M.E.S.; NAVES.J.A.M. Aspectos da Biologia de Lutzomyia do Complexo squamiventris (Lutz \& Neiva, 1912) (Diptera, Psychodidae) em Condiçðes Naturais, na cidade de Goiânia-Goiás. Rev.Pat.Trop.,23 ( 2 ):229-234,jul./dez.1994

\section{REFERÊNCIAS BIBLIOGRÁFICAS}

01. BARRETO,M.P. Uma nova espécie de flebótomo do estado de Goiás, Brasil, e chave para determinação das espécies afins. (Diptera,Psychodidae) Rev.Bras.Biol. 6(3): 427-434,1946.

02. CARVALHO,M.E.S.D; LUSTOSA,M.E.S.C. \& NAVES,H.A.M. Contribuição ao conhecimento da fauna flebotomínica do estado de Goiás e Distrito Federal II. 1986-1987. Rev.Pat.Trop. 18(1):7-14, 1989.

03. CARVALHO.M.E.S.D; NAVES,H.A.M. \& COSTA,J.A.da. Outras espécies de Lutzomyia (França, 1924) (Diptera,Psychodidae) em Goiânia-Goiás. Rev.Pat.Trop. 22 (1): 105, 1993.

04. CARVALHO, M.E.S.D; NAVES,H.AM. \& LUSTOSA,E. de S. Ocorrência de Lutzomyia flaviscutellata (LUTS \& NEIVA, 1912) (Diptera, Psychodidae) em Goiânia-Goiás, Brasil. Rev.Pat.Trop.20 (2): 207-210, 1991.

05. COELHO,M.V; CUNHA, A.S.; FALCÃO,A.R. - Notas sobre um foco de Calazar no sudoeste do Estado de Goiás. Rev.Brasil.Malariol. Doenças Trop.,17: 143-148, 1965.

06. FORATTINI,O.P. Entomologia Médica.São Paulo, Universidade de São Paulo, 4, $1973,658 \mathrm{p}$.

07. GALATI,E.A.B. Sistemática dos Phlebotominae (Diptera,Psychodidae) das Américas. 1990. 200p. Tese de Doutoramento apresentada ao Departamento de Epidemiologia da Faculdade de Saúde Pública da USP.

08. LUSTOSA,E.de S.;NAVES,H.A.M \& CARVALHO,M.E.S.D.; BARBOSA,W. Contribuição ao conhecimento da fauna flebotomínica do estado de Goiás. 1984-1985. Nota prévia I. Rev.Pat.Trop.15 (1):7-11, 1986.

09. MARTINS,A.V.; WILLIANS,P. \& FALCÃO, A.L. American sandflies. (Diptera, Psychodidae). Ed. Academia Brasileira de Ciências, 195p., 1978. 
CARVALHO,M.E.S.; NAVES.J.A.M. Aspectos da Biologia de Lutzomyia do Complexo squamiventris (Lutz \& Neiva, 1912) (Diptera, Psychodidae) em Condiçð̃es Naturais, na cidade de Goiânia-Goiás. Rev.Pat.Trop.,23 ( 2):229-234,jul./dez.1994

10. MARTINS,A.V.; FALCÃO,A.L. \& SILVA,J.E. Descrição da fêmed de Lutzomyia teratodes (Martins,Falcão \& Silva 1964 - Diptera, Psychodidae, Phlebotominae). Rev.Brasil.Biol.35:(3), 515-517, 1975.

11. MARTINS,A.V.; FALCÃO,A.L. \& SILVA,J.E. Nota sobre os flebótomos do Estado de Goiás, com a descrição de duas espécies novas e da fêmea de Lutzomyia longipennis (Barreto,1946) e redescrição do macho de L.evandroi (Costa Lima \& Antunes, 1936) (Diptera,Psychodidae). Rev.Brasil.Malariol. Doenças Trop.14: 379-394,1962.

12. MARTINS,A.V.; FALCÃO,A.L. \& SILVA,J.E. Um novo flebótomo do estado de Goiás, Lutzomyia teratodes sp.n .( Diptera,Psychodidae ). Rev.Brasil.Biol.24 (3); 321-324, 1964.

13. NAVES,H.A.M.; CARVALHO,M.E.S.D. \& CARNEIRO,E. Ocorrência de Lutzomyia do complexo squamiventris (Lutz \& Neiva,1912), e Lutzomyia fischeri ( Pinto, 1926) (Diptera, Psychodidae ) ,em Goiânia Goiás.Rev.Pat.Trop.22 (1): 97 Jan./Jun.1993.

14. NAVES,H.A.M.; CARVALHO,M.E.S.D. \& LUSTOSA,E.de S. Ocorrência de Lutzomyia intermedia (Lutz \& Neiva, 1912) (Diptera, Psychodidae) em Goiânia-Goiás, Brasil. Rev.Pat.Trop.20 (2): 207-210, 1991.

15. NAVES,H.A.M.; CARVALHO,M.E.S.D. \& LUSTOSA, E. de S. Ocorrência de Lutzomyia whitmani (Antunes \& Coutinho, 1939) (Diptera, Psychodidae) em Goiânia-Goiás. In: SEMINÁRIO EM PATOLOGIA TROPICAL E SAÚDE PÚBLICA.1. Goiânia.1992. Rev.Pat.Trop., 21 (1): Jan./Jun. Suplemento. 1992.

16. RYAN,L. Flebótomos do Estado do Pará, Brasil. Belém. Documento Técnico No.1), 1986.

17. YOUNG, D.G. \& FAIRCHILD,G.B. Studies of Phlebotominae sandflies. Flórida. Annual Report. 1974. 